\title{
Control of Ultracold Collisions with Frequency-Chirped Light
}

\author{
M. J. Wright, ${ }^{1}$ S. D. Gensemer, ${ }^{2}$ J. Vala, ${ }^{3}$ R. Kosloff, ${ }^{4}$ and P. L. Gould ${ }^{1}$ \\ ${ }^{1}$ Department of Physics, U-3046, University of Connecticut, Storrs, Connecticut 06269, USA \\ ${ }^{2}$ Van der Waals-Zeeman Institut, Universiteit van Amsterdam, Valckenierstraat 65, 1018 XE Amsterdam, The Netherlands \\ ${ }^{3}$ Department of Chemistry and Pitzer Center for Theoretical Chemistry, University of California, Berkeley, California 94720, USA \\ ${ }^{4}$ Department of Physical Chemistry and the Fritz. Haber Research Center for Molecular Dynamics, the Hebrew University, \\ 91094 Jerusalem, Israel
}

(Received 24 September 2004; published 1 August 2005)

\begin{abstract}
We report on ultracold atomic collision experiments utilizing frequency-chirped laser light. A rapid chirp below the atomic resonance results in adiabatic excitation to an attractive molecular potential over a wide range of internuclear separation. This leads to a transient inelastic collision rate which is large compared to that obtained with fixed-frequency excitation. The combination of high efficiency and temporal control demonstrates the benefit of applying the techniques of coherent control to the ultracold domain.
\end{abstract}

The ability to control the dynamics of microscopic systems has been a major motivation in physics and chemistry in recent years [1]. The development of ultrafast lasers and pulse-shaping techniques has allowed selective bond breaking and coherent control of chemical reactions [2]. At the other temporal extreme, slow collisions between ultracold atoms [3,4] can be controlled by long-range laser excitation because the colliding atoms have minimal kinetic energy and their trajectories are easily manipulated. In the present work, we adapt the techniques of coherent control to the nanosecond time scale and use frequencychirped light to control inelastic collisions between ultracold atoms. The combination of adiabatic excitation and the large number of atom pairs addressed by the chirp leads to a large transient collision rate. Such extensions of coherent control to the ultracold domain may significantly benefit processes such as ultracold molecule formation $[5,6]$. As an example, chirped two-photon Raman photoassociation $[7,8]$ may provide an attractive alternative to magnetic Feshbach resonances for the coherent conversion of an atomic Bose-Einstein condensate into a molecular one. Because light can be controlled much faster than magnetic fields, the chirped excitation techniques developed here may allow the probing of quantum gases on much shorter time scales than previously achieved [9].

There have been a number of experiments exploring the temporal dynamics of ultracold collisions. A long-lived ground-state shape resonance has been probed by timedependent photoassociation [10,11], and laser-induced collisions have been followed in real time [12,13]. Motivated by earlier predictions [14], photoassociative ionization dynamics on the nanosecond time scale have been probed with picosecond pulses [15]. In ultracold highly excited Rydberg atoms, the microsecond-scale evolution of resonant energy transfer has been observed via field ionization $[16,17]$. Our use of rapidly frequency-chirped laser light brings a new dimension to these studies. The temporal evolution of the light can lead to an efficient and robust adiabatic transfer of population to the excited state. Also, the wide range of frequencies spanned by the chirp leads to excitation of atom pairs over a wide range of internuclear separations. The advantages of chirped excitation have been discussed in the context of ultracold atom photoassociation with picosecond pulses [18-20].

The basic idea of the experiment is shown in Fig. 1. Laser light is chirped over a range of frequencies below the atomic resonance, exciting pairs of trapped atoms to the $C_{3} / R^{3}$ attractive molecular potential at various internuclear separations $R$. If the resulting attraction imparts

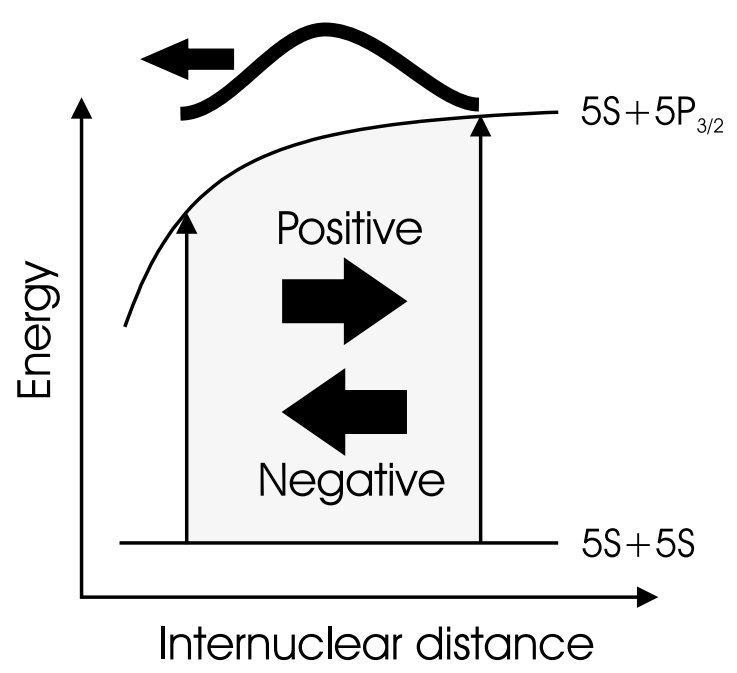

FIG. 1. Schematic of chirped collision experiment. The longrange ground-state $(5 S+5 S)$ and excited-state $\left(5 S+5 P_{3 / 2}\right)$ molecular potentials are shown, as well as the frequency-chirped (positive and negative) light that drives the transition between them. The chirp creates an excited-state wave packet that subsequently rolls down the attractive potential. In this example, the chirp extends from 800 to $100 \mathrm{MHz}$ below the asymptote, exciting atom pairs to the $0_{u}^{+}$potential over the range $R=$ $517 a_{0}$ to $1034 a_{0}$. 
sufficient kinetic energy (e.g., $1 \mathrm{~K}$ per atom) before spontaneous emission occurs, the atom pair will be ejected from the trap [21,22]. Since the atoms are ultracold (e.g., $50 \mu \mathrm{K})$, their motion on the ground state is minimal on the nanosecond time scale of the chirp.

The effectiveness of the chirped excitation is illustrated in the calculations shown in Fig. 2. The initial state for the colliding ${ }^{85} \mathrm{Rb}$ atoms is assumed to be a ground-state wave packet centered at $1500 a_{0}\left(a_{0}=5.29 \times 10^{-9} \mathrm{~cm}\right)$. Its width, $211 a_{0}$, is uncertainty limited according to the spread of relative velocities at a temperature of $50 \mu \mathrm{K}$ [18]. The assumed temporal dependences of the frequency and intensity of the chirped light are shown in Figs. 2(c) and 2(b), respectively. In Fig. 2(a), the calculated transfer of population $[18,23]$ from the ground state to the $0_{u}^{+}$excited-state potential [22], neglecting hyperfine structure, is shown. Note that the center of the wave packet is resonant at a chirp detuning $\Delta=-32.8 \mathrm{MHz}$. Efficient excitation occurs via a combination of (1) off-resonant transfer when the intensity is high ( $t \approx 50 \mathrm{~ns})$, and ( 2 ) adiabatic passage when the center of the wave packet passes through reso-

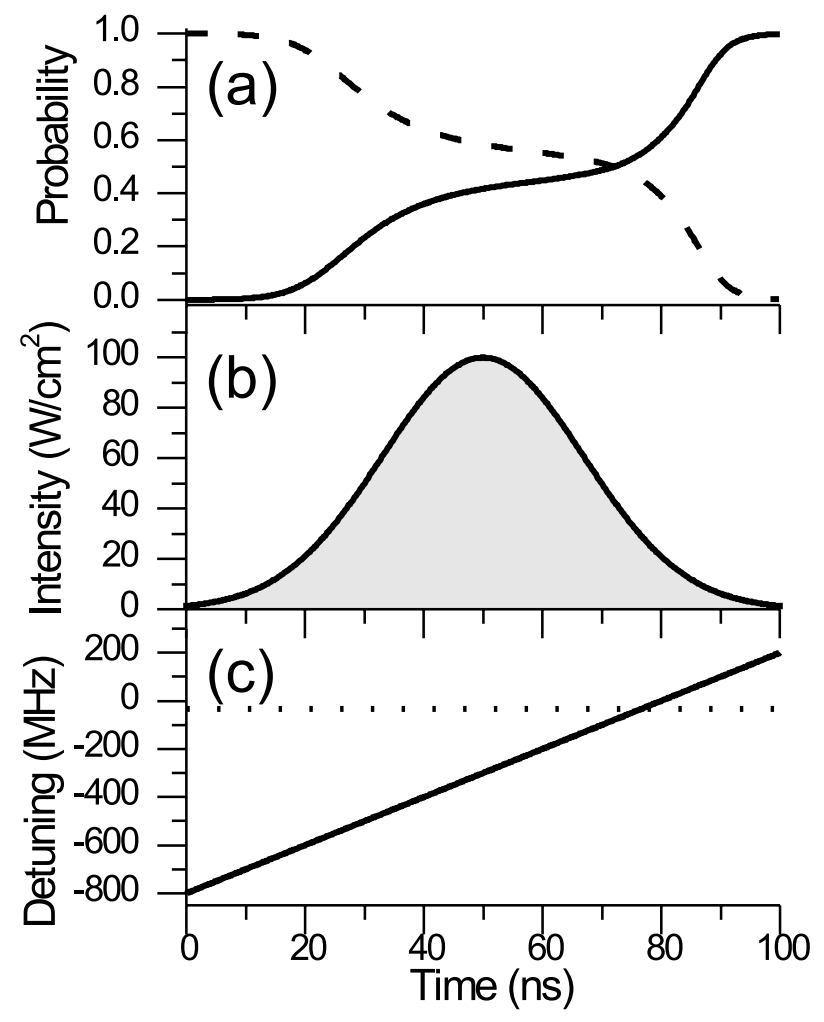

FIG. 2. Time dependence of excitation for a positively chirped pulse calculated using the Schrödinger equation. The detuning $\Delta$ relative to the asymptote is shown in (c), the intensity of the Gaussian pulse in (b), and the ground-state (dashed line) and excited-state $0_{u}^{+}$(solid line) populations in (a). An uncertaintylimited initial wave packet centered at $1500 a_{0}$ is assumed and spontaneous emission is ignored. The dotted horizontal line in (c) indicates the frequency resonant with the peak of the wave packet. nance ( $t \approx 75 \mathrm{~ns}$ ). In this example, a positive chirp is chosen. For the parameters of Fig. 2, a negative chirp gives similar results for the final excitation probability because the atomic motion is slow on the time scale of the chirp. However, where the potential is steeper and the atoms accelerate more rapidly, the positive chirp is expected to be more robust and efficient. This is because the positively chirped excitation proceeds from smaller to larger $R$, while the motion of the atom pair on the attractive potential proceeds oppositely. Therefore, an atom pair, once excited, is unlikely to further interact with the chirped field, minimizing the possibility of stimulated emission back to the ground state [23-25].

The experiment is performed by measuring the inelastic collisional rate constant $\beta$ for ultracold ${ }^{85} \mathrm{Rb}$ atoms in a magneto-optical trap (MOT) [26]. A trap-loss collision occurs when the atom pair arrives at short range (e.g., $R<$ $\left.140 a_{0}\right)$ in the excited state. The rate constant for these inelastic collisions is extracted from the density-dependent decay of the trapped sample [27]; i.e., the collisional loss rate per atom is $\beta n$, where $n$ is the atomic density. The MOT is operated in the phase-stable configuration [28] in order to reduce fluctuations in the parameters of the trapped cloud. The axial magnetic field gradient is $12 \mathrm{G} / \mathrm{cm}$, the total central intensity (sum of all six beams) is $21.6 \mathrm{~mW} / \mathrm{cm}^{2}$, and the detuning is $-2 \Gamma$ relative to the $5 S_{1 / 2}(F=3) \rightarrow 5 P_{3 / 2}\left(F^{\prime}=4\right)$ cycling transition, where $\Gamma=2 \pi(5.9 \mathrm{MHz})$ is the natural linewidth. A slow beam generated from a second MOT [29] is used for loading, allowing low background pressures $\left(\approx 10^{-10}\right.$ torr $)$ and long MOT lifetimes $(\approx 60 \mathrm{~s})$.

The frequency-chirped light, with chirp rates up to $15 \mathrm{GHz} / \mu \mathrm{s}$, is produced by rapidly ramping the injection current of an external-cavity diode laser. In order to obtain higher power and minimize the amplitude modulation, a small fraction of this master laser's chirped output is used to injection lock a separate slave diode laser [30]. The linearly polarized slave laser output is focused to a diameter of $\approx 100 \mu \mathrm{m}$, approximately matching the size of the trapped-atom sample. Peak intensities up to $100 \mathrm{~W} / \mathrm{cm}^{2}$ are thus obtained. This technique for frequency control on the ns time scale is well suited to the dynamics of ultracold atoms interacting at long range.

The timing of the experiment is controlled with acoustooptical modulators (AOMs). Every $\tau_{r}=722 \mu \mathrm{s}$, the MOT light is switched off for $240 \mu \mathrm{s}$, during which time a number of pulses $N_{c}$ (typically 60) of chirped light is applied. The time-averaged number of chirps per second is thus given by $\nu_{c}=N_{c} / \tau_{r}$ and is typically $8.3 \times 10^{4} \mathrm{~s}^{-1}$. The master laser is continuously chirped with a linear ramp at a typical frequency of $2 \mathrm{MHz}$, yielding successive chirps separated by $500 \mathrm{~ns}$. The desired frequency range of each chirp is selected with a 40 ns FWHM AOM pulse which is synchronized with the chirp ramp. The repumping light of the MOT is left on continuously in order to correct any 
optical pumping that may occur during the sequence of chirped excitations.

The MOT light itself causes a background level of traploss collisions that must be subtracted out in order to isolate the rate due to the chirped light. This background and the desired signal are comparable in size, indicating the importance of MOT stability. The absolute atomic density is determined by combining the calibrated atomic fluorescence and the volume of the MOT cloud as measured with a CCD camera. Increased fluorescence due to free-atom excitation by the chirp is accounted for in calculating the collisional rate constant. This increase is $<10 \%$ of the time-averaged MOT fluorescence. With all other parameters fixed, the number of chirps occurring during each MOT-off window was varied (from 0 to 70 ) and the expected linear dependence of the collision rate was verified.

The intensity dependence of $\beta$ for both positive and negative chirps is shown in Fig. 3. In the high intensity (adiabatic) limit, we observe the expected saturation of the chirp-induced excitation. Also shown are the theoretical curves based on a Monte Carlo simulation, which incorporates the calculated excitation probabilities [18,23], as shown in Fig. 2, for wave packets centered at different values of $R$. These excitation probabilities include averaging over the angle between the collision axis and the laser polarization direction. In the simulation, an initial atompair separation and two-dimensional relative velocity are chosen according to the appropriate distributions. The probability of excitation by the chirp is then calculated. If excitation occurs, the trajectory on the attractive poten-

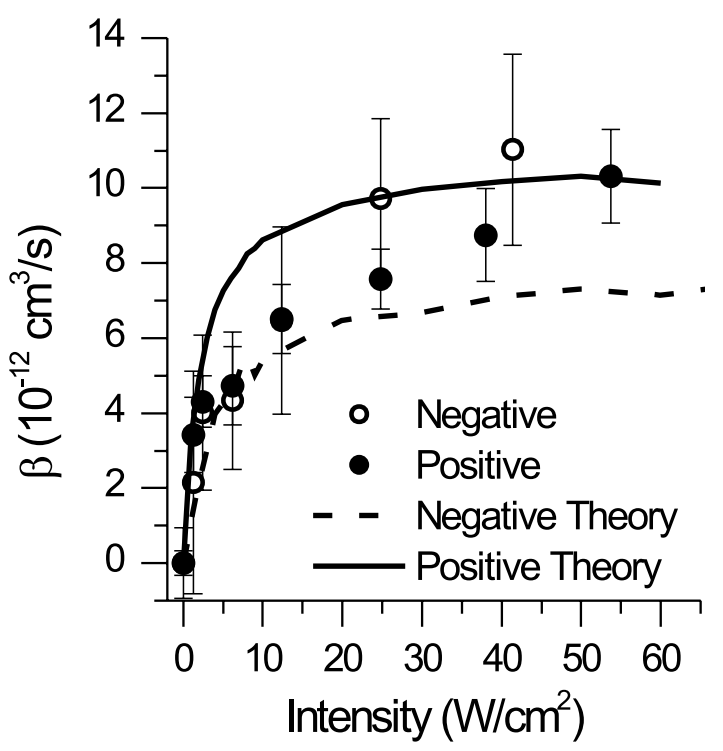

FIG. 3. Intensity dependence of the collisional rate constant for both positive and negative chirps. The chirp rate is $10 \mathrm{GHz} / \mu \mathrm{s}, \quad \nu_{c}=8.3 \times 10^{4} \mathrm{~s}^{-1}$, and the $40 \mathrm{~ns}$ pulses are centered at $\Delta=-300 \mathrm{MHz}$. Also shown are theoretical curves based on the Monte Carlo simulation described in the text. These curves are scaled vertically by 0.66 to match the data. tial (assumed to be $0_{u}^{+}$) is followed to see if the pair reaches short range before spontaneous emission occurs. Averaging over initial separation, velocity, excitation efficiency, and the time of spontaneous emission gives the number of collisions per atom per chirp. Multiplying this by the chirp repetition rate and dividing by the assumed density yields the time-averaged collisional rate constant. The calculation is scaled vertically by a factor of 0.66 , but the intensity dependence is seen to match the data quite well. The simulations predict somewhat smaller values of $\beta$ for the negative chirp, while the experiment shows no significant difference. Our assumption of a single potential is certainly an oversimplification relative to the experiment.

A key point to verify is that the excitation of colliding atom pairs depends on the chirp rate and not simply on the fact that a range of frequencies is present. Unfortunately, the interdependence of the various chirp parameters prevents a straightforward comparison. For example, if we increase the chirp rate, while keeping the pulse width fixed, then the range of the chirp will increase. If we compensate by reducing the pulse width, then the energy per pulse (and thus the time-averaged laser intensity) will decrease. Instead, we have chosen to compare two extremes: a fast chirp and a quasistatic averaging over the chirp range. The timing diagrams for these two situations are shown in the Fig. 4 insets. For the fast chirp, the pulses are synchronized with the chirp ramps so each pulse includes a complete

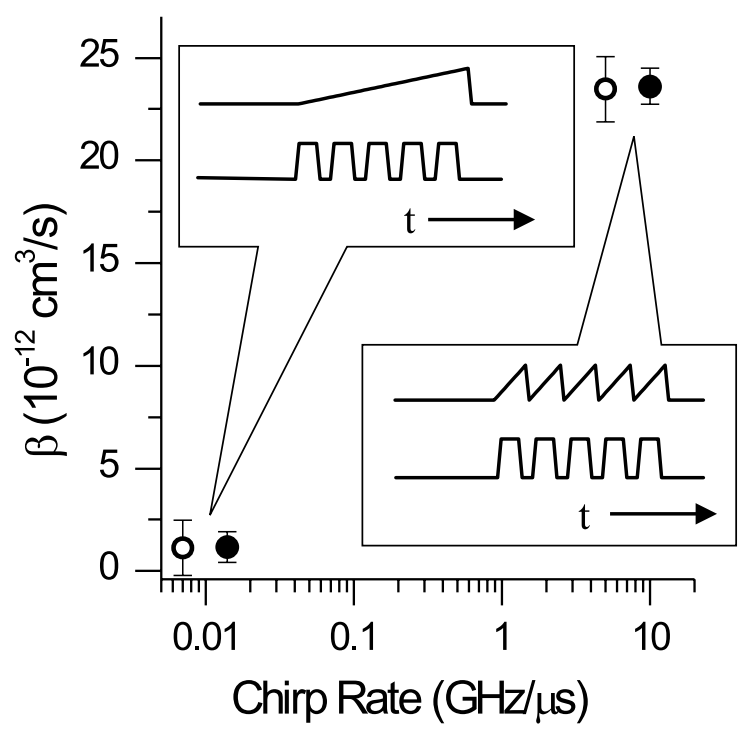

FIG. 4. Collision rate constant as a function of chirp rate. The intensity and pulse width are fixed at $50 \mathrm{~W} / \mathrm{cm}^{2}$ and $40 \mathrm{~ns}$, respectively, $\nu_{c}=20 \times 10^{4} \mathrm{~s}^{-1}$, and each chirp ranges from -800 to $+200 \mathrm{MHz}$. The negative chirp points (open symbols) have been offset horizontally for clarity. The insets show the timing of the pulses (lower trace) and the positive chirp (upper trace). For the fast chirp, each pulse corresponds to a complete chirp. For the slow chirp, each pulse samples a small portion of the chirp, which is equivalent to a quasistatic averaging over the frequency range of the chirp. 
chirp. For the quasistatic case, a single slow chirp occurs during the train of pulses. The pulses are not synchronized with the chirp ramp, so when averaged over many cycles, all frequencies of the ramp are sampled. Note that the number of pulses, the time-averaged intensity, and the range of frequencies sampled are the same for the two cases. As the data shows, for both the positive and the negative chirps, the fast chirp is at least an order of magnitude more efficient in causing collisions.

We can estimate the efficiency of the fast chirp data by comparing with the Monte Carlo simulation described above. Values for the $C_{3}$ coefficients and $R$-dependent lifetimes from [22] are used, and the effects of excitedstate hyperfine structure are ignored. For the positive chirp case and the parameters of Fig. 4, the calculated rate constants for the various attractive potentials at the $5 S+$ $5 P_{3 / 2}$ asymptote are (in units of $10^{-12} \mathrm{~cm}^{3} \mathrm{~s}^{-1}$ ) 37 for $0_{u}^{+}$, 42 for $1_{g}, 12$ for $0_{g}^{-}$, and 33 for $1_{u}$. For the negative chirp, the calculations yield 27 for $0_{u}^{+}, 29$ for $1_{g}, 6$ for $0_{g}^{-}$, and 23 for $1_{u}$. We have not included the $2_{u}$ state because its excitation and decay are asymptotically forbidden. The measured values of 24 are known only to within a factor of 2 due to uncertainties in the absolute atomic density. Nevertheless, the comparison indicates that the chirped excitation, for both positive and negative chirps, is quite efficient at exciting all atom pairs within the spherical shell addressed by the chirp. The inner radius of this shell is determined by the initial (final) frequency of the positive (negative) chirp. Since we chirp through the atomic resonance (corresponding to $R=\infty$ ), the outer radius is limited by survival of excitation to short range [21,22].

In summary, we have used pulses of nanosecond-scale frequency-chirped light to efficiently induce ultracold collisions over a wide range of internuclear separations. Future work will explore the dependence on chirp range and possible cooperative effects between successive chirps. Detailed temporal control of the frequency and intensity should be possible, allowing optimization via feedback from learning algorithms, as successfully demonstrated with femtosecond pulses [2]. A particularly intriguing application of such coherent control to the ultracold domain would be the use of shaped collisional wave packets in ultracold ground-state molecule formation.

This work was supported in part by the Chemical Sciences, Geosciences and Biosciences Division, Office of Basic Energy Sciences, Office of Science, U.S. Department of Energy.
[1] S. A. Rice and M. Zhao, Optimal Control of Molecular Dynamics (Wiley, New York, 2000); M. Shapiro and P. Brumer, Principles of Quantum Control of Molecular Processes (Wiley, New York, 2003), and references therein.

[2] R. S. Judson and H. Rabitz, Phys. Rev. Lett. 68, 1500 (1992); A. Assion et al., Science 282, 919 (1998).

[3] J. Weiner et al., Rev. Mod. Phys. 71, 1 (1999).

[4] J. Weiner, Cold and Ultracold Collisions in Quantum Microscopic and Mesoscopic Systems (Cambridge University Press, New York, 2003).

[5] J. T. Bahns, P. L. Gould, and W. C. Stwalley, Adv. At. Mol. Opt. Phys. 42, 171 (2000).

[6] F. Masnou-Seeuws and P. Pillet, Adv. At. Mol. Opt. Phys. 47, 53 (2001).

[7] J. Javanainen and M. Mackie, Phys. Rev. A 59, R3186 (1999).

[8] D. J. Heinzen et al., Phys. Rev. Lett. 84, 5029 (2000).

[9] J. M. Gerton et al., Nature (London) 408, 692 (2000).

[10] H. Boesten et al., Phys. Rev. Lett. 77, 5194 (1996).

[11] H. Boesten et al., J. Phys. B 32, 287 (1999).

[12] S. D. Gensemer and P. L. Gould, Phys. Rev. Lett. 80, 936 (1998).

[13] C. Orzel et al., Phys. Rev. Lett. 80, 5093 (1998).

[14] M. Machholm, A. Guisti-Suzor, and F. Mies, Phys. Rev. A 50, 5025 (1994).

[15] F. Fatemi et al., Phys. Rev. A 64, 033421 (2001).

[16] R.A.D.S. Zanon et al., Phys. Rev. A 65, 023405 (2002).

[17] A. L. De Oliveira et al., Phys. Rev. Lett. 90, 143002 (2003).

[18] J. Vala et al., Phys. Rev. A 63, 013412 (2001).

[19] E. Luc-Koenig et al., Phys. Rev. A 70, 033414 (2004).

[20] E. Luc-Koenig, M. Vatasescu, and F. Masnou-Seeuws, Eur. Phys. J. D 31, 239 (2004).

[21] A. Gallagher and D. E. Pritchard, Phys. Rev. Lett. 63, 957 (1989).

[22] P. S. Julienne and J. Vigue, Phys. Rev. A 44, 4464 (1991).

[23] J. Vala and R. Kosloff, Opt. Express 8, 238 (2001).

[24] J. Cao, C. J. Bardeen, and K. R. Wilson, Phys. Rev. Lett. 80, 1406 (1998).

[25] J. Cao, C. J. Bardeen, and K. R. Wilson, J. Chem. Phys. 113, 1898 (2000).

[26] E. L. Raab et al., Phys. Rev. Lett. 59, 2631 (1987).

[27] S. D. Gensemer et al., Phys. Rev. A 56, 4055 (1997).

[28] A. Rauschenbeutel et al., Opt. Commun. 148, 45 (1998).

[29] Z. T. Lu et al., Phys. Rev. Lett. 77, 3331 (1996).

[30] M. J. Wright, S. D. Gensemer, and P. L. Gould, Rev. Sci. Instrum. 75, 4718 (2004). 